\title{
THE 3-DIMENSIONAL CORED AND LOGARITHM POTENTIALS: PERIODIC ORBITS
}

\author{
MAITÉ KULESZA ${ }^{1}$ AND JAUME LLIBRE ${ }^{2}$
}

\begin{abstract}
We study analytically families of periodic orbits for the cored and logarithmic Hamiltonians $H\left(x, y, z, p_{x}, p_{y}, p_{z}\right)=\left(p_{x}^{2}+p_{y}^{2}+p_{z}^{2} / q\right) / 2+\left(1+x^{2}+\right.$ $\left.\left(y^{2}+z^{2}\right) / q^{2}\right)^{1 / 2}$, and $H\left(x, y, z, p_{x}, p_{y}, p_{z}\right)=\left(p_{x}^{2}+p_{y}^{2}+p_{z}^{2} / q\right) / 2+\left(\log \left(1+x^{2}+\right.\right.$ $\left.\left.\left(y^{2}+z^{2}\right) / q^{2}\right)\right) / 2$, with 3 degrees of freedom, which are relevant in the analysis of the galactic dynamics. First, after introducing a scale transformation in the coordinates and momenta with a parameter $\varepsilon$, we show that both systems give essentially the same set of equations of motion up to first order in $\varepsilon$. Then the conditions for finding families of periodic orbits, using the averaging theory up to first order in $\varepsilon$, apply equally to both systems in every energy level $H=h>0$. The averaging method used proves the existence of at most three periodic orbits, for $\varepsilon$ small enough, and gives an analytic approximation for the initial conditions of these periodic orbits.
\end{abstract}

\section{INTRODUCTION}

In this paper we are interested in 3 degrees Hamiltonian systems of the form

$$
H\left(x, y, z, p_{x}, p_{y}, p_{z}\right)=\frac{1}{2}\left(p_{x}^{2}+p_{y}^{2}+\frac{p_{z}^{2}}{q}\right)+V\left(x^{2}, y^{2}, z^{2}\right),
$$

where $V$ a smooth potential with an absolute minimum and a reflection symmetry with respect the three axes. The motivation for the choice of these symmetries becomes from the interest of these potentials in galactic dynamics. In particular, we considered the cored potential

$$
V_{C}=\sqrt{1+x^{2}+\frac{y^{2}+z^{2}}{q^{2}}}
$$

and the logarithm potential

$$
V_{L}=\frac{1}{2} \log \left(1+x^{2}+\frac{y^{2}+z^{2}}{q^{2}}\right) .
$$

such potentials in 2 degrees of freedom have been studied by several authors, see for instance, $[1,3,4,6,7,8]$.

Our goal is to study the periodic orbits of the corresponding Hamiltonian differential system using the averaging theory.

Belmont et al. [8] applied the method of resonant detuned normal forms to investigate properties of the logarithmic galactic potential. These forms are obtained

2010 Mathematics Subject Classification. Primary 34C10, 34C25.

Key words and phrases. periodic orbits, galactic potential, cored potential, logarithm potential, averaging theory. 
with a method based on the Lie transformation which is analogous to the Prendergast method applied by Contopoulos and Seimenis. In Pucacco et al. [7], they showed that it is possible to find periodic orbits with this method.

The averaging method has already been applied to others particular galactic potentials. Jiménez-Lara and Llibre [3] obtained two families of periodic orbits in the plane, when the parameter $q$ is irrational. Lacomba and Llibre [4] studied the potential with a general perturbation of fourth order and obtained four families of periodic orbits in the plane, when the parameter $q$ is equal a 1 . The parameter $q$ gives the ellipticity of the potential, which ranges in the interval $[0.6,1]$.

In this paper, we find new families of periodic orbits parameterized by the energy and depending on the parameter $q$.

\section{Statement of THE PROBlem}

We shall study the periodic orbits of the Hamiltonians systems associated to the

$$
\begin{gathered}
H_{C}=\frac{1}{2}\left(p_{x}^{2}+p_{y}^{2}+\frac{p_{z}^{2}}{q}\right)+\sqrt{1+x^{2}+\frac{y^{2}+z^{2}}{q^{2}}} \\
H_{L}=\frac{1}{2}\left(p_{x}^{2}+p_{y}^{2}+\frac{p_{z}^{2}}{q}\right)+\frac{1}{2} \log \left(1+x^{2}+\frac{y^{2}+z^{2}}{q^{2}}\right),
\end{gathered}
$$

called the cored and logarithm Hamiltnonians, respectively.

The cored Hamiltonian system is

$$
\begin{aligned}
\dot{x}=\frac{\partial H_{C}}{\partial p_{x}} & =p_{x}, \\
\dot{y}=\frac{\partial H_{C}}{\partial p_{y}} & =p_{y}, \\
\dot{z}=\frac{\partial H_{C}}{\partial p_{z}} & =\frac{p_{z}}{q}, \\
\dot{p}_{x}=-\frac{\partial H_{C}}{\partial x} & =-\frac{x}{\sqrt{1+x^{2}+\frac{y^{2}+z^{2}}{q^{2}}}}, \\
\dot{p}_{y}=-\frac{\partial H_{C}}{\partial y} & =-\frac{q^{2} \sqrt{1+x^{2}+\frac{y^{2}+z^{2}}{q^{2}}}}{z} \\
\dot{p}_{z}=-\frac{\partial H_{C}}{\partial z} & =-\frac{q^{2} \sqrt{1+x^{2}+\frac{y^{2}+z^{2}}{q^{2}}}}{}
\end{aligned}
$$


and the logarithmic Hamiltonian system is

$$
\begin{aligned}
\dot{x} & =\frac{\partial H_{C}}{\partial p_{x}}=p_{x} \\
\dot{y} & =\frac{\partial H_{C}}{\partial p_{y}}=p_{y} \\
\dot{z} & =\frac{\partial H_{C}}{\partial p_{z}}=\frac{p_{z}}{q} \\
\dot{p}_{x}=-\frac{\partial H_{C}}{\partial x} & =-\frac{x}{1+x^{2}+\frac{y^{2}+z^{2}}{q^{2}}} \\
\dot{p}_{y}=-\frac{\partial H_{C}}{\partial y} & =-\frac{q^{2}\left(1+x^{2}+\frac{y^{2}+z^{2}}{q^{2}}\right)}{z} \\
\dot{p}_{z}=-\frac{\partial H_{C}}{\partial z} & =-\frac{q^{2}\left(1+x^{2}+\frac{y^{2}+z^{2}}{q^{2}}\right)}{}
\end{aligned}
$$

After introducing a non-canonical scale transformation with a small parameter $\varepsilon>0$,

$$
\left\{x, y, z, p_{x}, p_{y}, p_{z}\right\} \quad \rightarrow \quad\left\{\sqrt{\varepsilon} x_{1}, \sqrt{\varepsilon} y_{1}, \sqrt{\varepsilon} z_{1}, \sqrt{\varepsilon} p_{x_{1}}, \sqrt{\varepsilon} p_{y_{1}}, \sqrt{\varepsilon} p_{z_{1}}\right\} .
$$

Note that in what follows the six variables $\left(x_{1}, y_{1}, z_{1}, p_{x_{1}}, p_{y_{1}}, p_{z_{1}}\right)$ are denoted again by $\left(x, y, z, p_{x}, p_{y}, p_{z}\right)$ respectively. Thus both Hamiltonian systems (5) and (6) can be reduced to study the same differential system

$$
\begin{aligned}
\dot{x} & =p_{x}, \\
\dot{y} & =p_{y}, \\
\dot{z} & =\frac{p_{z}}{q}, \\
\dot{p}_{x} & =-x+\varepsilon \frac{x\left(q^{2} x^{2}+y^{2}+z^{2}\right)}{2 q^{2}}+\mathcal{O}\left(\varepsilon^{2}\right), \\
\dot{p}_{y} & =-\frac{y}{q^{2}}+\varepsilon \frac{x\left(q^{2} x^{2}+y^{2}+z^{2}\right)}{2 q^{4}}+\mathcal{O}\left(\varepsilon^{2}\right), \\
\dot{p}_{z} & =-\frac{z}{q^{2}}+\varepsilon \frac{z\left(q^{2} x^{2}+y^{2}+z^{2}\right)}{2 q^{4}}+\mathcal{O}\left(\varepsilon^{2}\right) .
\end{aligned}
$$

This is the cored Hamiltonian system. The logarithm Hamiltonian system has the small modification that, instead of $\varepsilon$, it has $2 \varepsilon$. Then we proceed the study of the system (7) which includes both Hamiltonian systems.

We summarize our main results as follows.

Theorem 1. If $q, q^{1 / 2}$ and $q^{3 / 2}$ are irrational, then for $\varepsilon>0$ sufficiently small, at every energy level $H=h>0$ the perturbed differential system (7) has at least three periodic solution $\gamma^{k}(t, \varepsilon)=\left(x^{k}(t, \varepsilon), y^{k}(t, \varepsilon), z^{k}(t, \varepsilon), p_{x}^{k}(t, \varepsilon), p_{y}^{k}(t, \varepsilon), p_{z}^{k}(t, \varepsilon)\right)$, for $k=1,2,3$ such that

(i) $\gamma^{1}(0, \varepsilon) \longrightarrow(\sqrt{2 h}, 0,0,0,0,0)$ when $\varepsilon \rightarrow 0$; 
(ii) $\gamma^{2}(0, \varepsilon) \longrightarrow(0,2 \sqrt{h} q, 0,0,0,0)$ when $\varepsilon \rightarrow 0$;

(iii) $\gamma^{3}(0, \varepsilon) \longrightarrow(0,0, \sqrt{2 h} q, 0,0,0)$ when $\varepsilon \rightarrow 0$.

Moreover, the families of periodic solutions $\gamma^{1}(t, \varepsilon), \gamma^{2}(t, \varepsilon)$ and $\gamma^{3}(t, \varepsilon)$ bifurcate from planar periodic solutions of system (7) with $\varepsilon=0$ living in the planes $\left(x, 0,0, p_{x}, 0,0\right),\left(0, y, 0,0, p_{y}, 0\right)$ and $\left(0,0, z, 0,0, p_{z}\right)$, respectively.

Theorem 2. If $q$ is rational and $q^{3 / 2}$ is irrational, then for $\varepsilon>0$ sufficiently small, at every energy level $H=h>0$ the perturbed differential system (7) has at least two periodic solution $\gamma^{k}(t, \varepsilon)=\left(x^{k}(t, \varepsilon), y^{k}(t, \varepsilon), z^{k}(t, \varepsilon), p_{x}^{k}(t, \varepsilon), p_{y}^{k}(t, \varepsilon), p_{z}^{k}(t, \varepsilon)\right)$, for $k=1,2$ such that

(i) $\gamma^{1}(0, \varepsilon) \longrightarrow(\sqrt{2 h}, 0,0,0,0,0)$ when $\varepsilon \rightarrow 0$;

(ii) $\gamma^{2}(0, \varepsilon) \longrightarrow(0,0, \sqrt{2 h} q, 0,0,0)$ when $\varepsilon \rightarrow 0$.

Moreover, the families of periodic solutions $\gamma^{1}(t, \varepsilon)$ and $\gamma^{2}(t, \varepsilon)$ bifurcate from periodic solutions of system $(7)$ with $\varepsilon=0$ living in the planes $\left(x, 0,0, p_{x}, 0,0\right)$ and $\left(0,0, z, 0,0, p_{z}\right)$, respectively.

We remark that the periodic solutions $\gamma^{1}(t, \varepsilon)$ of Theorems 1 and 2 bifurcate from the same unperturbed periodic solution in each energy level $h$, but the results are different because in Theorem $1, q$ is irrational and in Theorem $2, q$ is rational.

Theorem 3. If $q$ is irrational and $q^{3 / 2}$ is rational, then for $\varepsilon>0$ sufficiently small, at every energy level $H=h>0$ the perturbed differential system (7) has at least one periodic solution $\gamma(t, \varepsilon)=\left(x(t, \varepsilon), y(t, \varepsilon), z(t, \varepsilon), p_{x}(t, \varepsilon), p_{y}(t, \varepsilon), p_{z}(t, \varepsilon)\right)$ such that $\gamma(0, \varepsilon) \longrightarrow(0, \sqrt{2 h} q, 0,0,0,0)$ when $\varepsilon \rightarrow 0$. Moreover, the families of periodic solutions $\gamma(t, \varepsilon)$ bifurcates from periodic solutions of system (7) with $\varepsilon=0$ living in the plane $\left(0, y, 0,0, p_{y}, 0\right)$.

We cannot study using the averaging theory if from the families of periodic solutions living in the space $\left(x, 0, z, p_{x}, 0, p_{z}\right)$ of system (7) with $\varepsilon=0$ under the assumptions of Theorem 3 bifurcate to $\varepsilon>0$ same families of periodic solutions. The problem is that we cannot compute some integrals which appear when applying the averaging method.

On the other hand, the case that remains also not covered by Theorems 1, 2 and 3 is the case $q$ and $q^{3 / 2}$ rationals. In this case, system (7) with $\varepsilon=0$ has a family of periodic solutions which fills the space $\left(x, y, z, p_{x}, p_{y}, p_{z}\right)$. But again in this case when we apply the averaging method appear integrals that we cannot compute.

We observe that the families of periodic solutions $\gamma^{1}$ and $\gamma^{2}$ of Theorem 1 , and the one of Theorem 3 have already appeared in the work [3] where this problem was studied with only 2 degrees of freedom.

\section{The Rescaled Differential SYSTEM}

The differential system (7) has the first integral

$$
H=\frac{1}{2}\left(p_{x}^{2}+p_{y}^{2}+\frac{p_{z}^{2}}{q^{2}}+x^{2}+\frac{y^{2}+z^{2}}{q^{2}}\right)-\varepsilon \frac{\left(q^{2} x^{2}+y^{2}+z^{2}\right)^{2}}{8 q^{4}}+\mathcal{O}\left(\varepsilon^{2}\right) .
$$


The solutions of the unperturbed equations (7) with $\varepsilon=0$ and arbitrary initial conditions $x(0)=x_{0}, y(0)=y_{0}, z(0)=z_{0}, p_{x}(0)=p_{x_{0}}, p_{y}(0)=p_{y_{0}}, p_{z}(0)=p_{z_{0}}$ are

$$
\begin{aligned}
x(t) & =x_{0} \cos t+p_{x_{0}} \sin t, \\
p_{x}(t) & =p_{x_{0}} \cos t-x_{0} \sin t, \\
y(t) & =y_{0} \cos \left(\frac{t}{q}\right)+p_{y_{0}} q \sin \left(\frac{t}{q}\right), \\
p_{y}(t) & =p_{y_{0}} \cos \left(\frac{t}{q}\right)-\frac{y_{0}}{q} \sin \left(\frac{t}{q}\right), \\
z(t) & =z_{0} \cos \left(\frac{t}{q^{3 / 2}}\right)+p_{z_{0}} q^{1 / 2} \sin \left(\frac{t}{q^{3 / 2}}\right), \\
p_{z}(t) & =p_{z_{0}} \cos \left(\frac{t}{q^{3 / 2}}\right)-\frac{z_{0}}{q^{1 / 2}} \sin \left(\frac{t}{q^{3 / 2}}\right) .
\end{aligned}
$$

Then for the unperturbed system the follows statements hold:

(a) If $q, q^{1 / 2}$ and $q^{3 / 2}$ are irrational, we have:

- one 2-parametric family of periodic orbits with period $2 \pi$ in the subspace $\left(x, 0,0, p_{x}, 0,0\right)$,

- one 2-parametric family of periodic orbits with period $2 \pi q$ in the subspace $\left(0, y, 0,0, p_{y}, 0\right)$, and

- one 2-parametric family of periodic orbits with period $2 \pi q^{3 / 2}$ in the subspace $\left(0,0, z, 0,0, p_{z}\right)$.

(b) If $q$ is rational and $q^{3 / 2}$ is irrational, we have:

- one 4-parametric family of periodic orbits with period $2 \pi q$ in the subspace $\left(x, y, 0, p_{x}, p_{y}, 0\right)$ and

- one 2-parametric family of periodic orbits with period $2 \pi q^{3 / 2}$ in the subspace $\left(0,0, z, 0,0, p_{z}\right)$.

(c) If $q$ is irrational and $q^{3 / 2}$ is rational, we have only one 2-parametric family of periodic orbits with period $2 \pi q$ in the subspace $\left(0, y, 0,0, p_{y}, 0\right)$.

\section{Proof of Theorem 1}

We consider the case $q, q^{1 / 2}$ and $q^{3 / 2}$ are irrational and we obtain three family of periodic orbits.

Proof of Theorem 1 for case $k=1$. We consider the unperturbed periodic solutions

$$
\begin{aligned}
x(t) & =x_{0} \cos t+p_{x_{0}} \sin t, \\
p_{x}(t) & =p_{x_{0}} \cos t-x_{0} \sin t, \\
y(t) & =0, \\
p_{y}(t) & =0, \\
z(t) & =0, \\
p_{z}(t) & =0 .
\end{aligned}
$$

in the plane $\left(x, 0,0, p_{x}, 0,0\right)$. Note that these periodic solutions living in the plane $\left(x, 0,0, p_{x}, 0,0\right)$ are not in resonance with the other periodic solutions of system $(7)$ for $\varepsilon=0$ living in the planes $\left(0, y, 0,0, p_{y}, 0,0\right)$ and $\left(0,0, z, 0,0, p_{z}\right)$ due to the fact 
that $q$ and $q^{3 / 2}$ are irrational. The first integral (8) when $\epsilon=0$ takes on these periodic solutions the value

$$
h=\frac{1}{2}\left(p_{x_{0}}^{2}+x_{0}^{2}\right) .
$$

We apply the First Order Averaging Theorem, see Theorem 4 of the appendix, to every fixed energy level, $H=h>0$. This allows to eliminate one of the coordinates, in this case $p_{x}$, and to reduce the study to dimension 5. Then $p_{x}$ at the energy level $H=h$ with $h$ given by (9) is

$$
\begin{aligned}
p_{x}= & \sqrt{p_{x_{0}}^{2}-p_{y}^{2}-\frac{p_{z}^{2}}{q}+x_{0}^{2}-x^{2}-\frac{y^{2}+z^{2}}{q^{2}}} \\
+ & \varepsilon \frac{\left(q^{2} x^{2}+y^{2}+z^{2}\right)^{2}}{8 q^{4} \sqrt{p_{x_{0}}^{2}-p_{y}^{2}-\frac{p_{z}^{2}}{q}+x_{0}^{2}-x^{2}-\frac{y^{2}+z^{2}}{q^{2}}}}+\mathcal{O}\left(\varepsilon^{2}\right),
\end{aligned}
$$

where we choose the plus sign for the determination of the square root, but the results that we shall obtain will be the same choosing the minus sign. This will be the case in all the proofs of this paper and we do not mention this in the next proofs.

The equations of motion (7) on the energy level $H=h>0$ are given by

$$
\begin{aligned}
\dot{x}= & \sqrt{p_{x_{0}}^{2}-p_{y}^{2}-\frac{p_{z}^{2}}{q}+x_{0}^{2}-x^{2}-\frac{y^{2}+z^{2}}{q^{2}}} \\
& +\varepsilon \frac{\left(q^{2} x^{2}+y^{2}+z^{2}\right)^{2}}{8 q^{4} \sqrt{p_{x_{0}}^{2}-p_{y}^{2}-\frac{p_{z}^{2}}{q}+x_{0}^{2}-x^{2}-\frac{y^{2}+z^{2}}{q^{2}}}+\mathcal{O}\left(\varepsilon^{2}\right),} \\
\dot{y}= & p_{y}, \\
\dot{p}_{y}= & -\frac{y}{q^{2}}+\varepsilon \frac{y\left(q^{2} x^{2}+y^{2}+z^{2}\right)}{2 q^{4}}+\mathcal{O}\left(\varepsilon^{2}\right), \\
\dot{z}= & \frac{p_{z}}{q}, \\
\dot{p}_{z}= & -\frac{z}{q^{2}}+\varepsilon \frac{z\left(q^{2} x^{2}+y^{2}+z^{2}\right)}{2 q^{4}}+\mathcal{O}\left(\varepsilon^{2}\right) .
\end{aligned}
$$

Now this differential system has the form $\dot{\mathbf{x}}=F_{0}(t, \mathbf{x})+\varepsilon F_{1}(t, \mathbf{x})+\varepsilon^{2} F_{2}(t, \mathbf{x}, \varepsilon)$, where $\mathbf{x}=\left(x, y, p_{y}, z, p_{z}\right)$,

$$
F_{0}\left(x, y, p_{y}, z, p_{z}\right)=\left(\sqrt{p_{x_{0}}^{2}-p_{y}^{2}-\frac{p_{z}^{2}}{q}+x_{0}^{2}-x^{2}-\frac{y^{2}+z^{2}}{q^{2}}}, p_{y},-y, \frac{p_{z}}{q},-\frac{z}{q^{2}}\right),
$$

and $F_{1}\left(x, y, p_{y}, z, p_{z}\right)$ is

$$
\left(\frac{\left(q^{2} x^{2}+y^{2}+z^{2}\right)^{2}}{8 q^{4} \sqrt{p_{x_{0}}^{2}-p_{y}^{2}-\frac{p_{z}^{2}}{q}+x_{0}^{2}-x^{2}-\frac{y^{2}+z^{2}}{q^{2}}}}, 0, \frac{y\left(q^{2} x^{2}+y^{2}+z^{2}\right)}{2 q^{4}}, 0, \frac{z\left(q^{2} x^{2}+y^{2}+z^{2}\right)}{2 q^{4}}\right) \text {. }
$$


Observe that the order of the variables is very important in the application of the Averaging Theorem (see Theorem 4 in the appendix). In what follows we use the notation introduced in the appendix. In this case we take the order $\left\{x, y, p_{y}, z, p_{z}\right\}$ and have $k=1, n=5, \alpha=x_{0}, \beta_{0}: \mathbb{R} \longrightarrow \mathbb{R}^{4}$ is $\beta_{0}(\alpha)=(0,0,0,0), \mathbf{z}_{\alpha}=\left(x_{0}, 0,0,0,0\right)$ is the initial condition of the unperturbed periodic orbits, for each $\mathbf{z}_{x_{0}}$ the solution $\mathbf{x}\left(t, \mathbf{z}_{x_{0}}\right)=\left(x_{0} \cos t+p_{x_{0}} \sin t, 0,0,0,0\right)$ is $2 \pi$-periodic, and $\xi\left(x, y, p_{y}, z, p_{z}\right)=x$.

Let $\mathbf{M}_{\mathbf{z}_{x_{0}}}(t)$ be the fundamental matrix satisfying $\mathbf{M}_{\mathbf{z}_{x_{0}}}(0)=\mathbf{I}$ solution of the variational equation (22) along the periodic solutions $\mathbf{x}\left(t, \mathbf{z}_{x_{0}}\right)$. Then $\mathbf{M}_{\mathbf{z}_{x_{0}}}(t)$ is given by

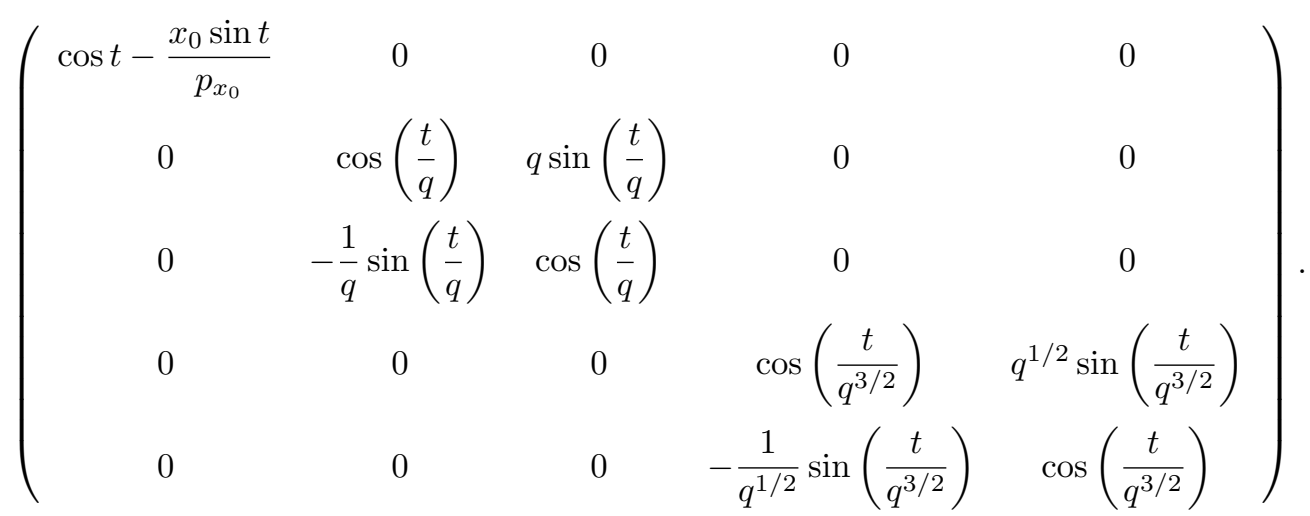

Now we verify the condition $\operatorname{det} \Delta_{x_{0}} \neq 0$, then we compute $\mathbf{M}_{\mathbf{z}_{x_{0}}}^{-1}(0)-\mathbf{M}_{\mathbf{z}_{x_{0}}}^{-1}(2 \pi)$ and we obtain

$$
\left(\begin{array}{ccccc}
0 & 0 & 0 & 0 & 0 \\
0 & 2 \sin ^{2}\left(\frac{\pi}{q}\right) & q \sin \left(\frac{2 \pi}{q}\right) & 0 & 0 \\
0 & -\frac{1}{q} \sin \left(\frac{2 \pi}{q}\right) & 2 \sin ^{2}\left(\frac{\pi}{q}\right) & 0 & 0 \\
0 & 0 & 0 & 2 \sin ^{2}\left(\frac{t}{q^{3 / 2}}\right) & q^{1 / 2} \sin \left(\frac{t}{q^{3 / 2}}\right) \\
0 & 0 & 0 & -\frac{1}{q^{1 / 2}} \sin \left(\frac{t}{q^{3 / 2}}\right) & 2 \sin ^{2}\left(\frac{t}{q^{3 / 2}}\right)
\end{array}\right)
$$

So

$$
\operatorname{det} \Delta_{x_{0}}=16 \sin ^{2}\left(\frac{\pi}{q^{3 / 2}}\right) \sin ^{2}\left(\frac{\pi}{q}\right) \neq 0 .
$$

The function $F_{1}$ along the periodic orbit is given by

$$
F_{1}\left(t, \mathbf{x}\left(t, \mathbf{z}_{x_{0}}\right)\right)=\left(\frac{\left(x_{0} \cos t+p_{x_{0}} \sin t\right)^{4}}{8\left(p_{x_{0}} \cos t-x_{0} \sin t\right)}, 0,0,0,0\right),
$$

then

$$
\mathbf{M}_{\mathbf{z}_{x_{0}}}^{-1}(t) F_{1}\left(t, \mathbf{x}\left(t, \mathbf{z}_{x_{0}}\right)\right)=\left(\frac{p_{x_{0}}\left(x_{0} \cos t+p_{x_{0}} \sin t\right)^{4}}{8\left(p_{x_{0}} \cos t-x_{0} \sin t\right)^{2}}, 0,0,0,0\right) .
$$

Thus, from (23) we have

$$
\mathcal{F}\left(x_{0}\right)=\int_{0}^{2 \pi} \frac{p_{x_{0}}\left(x_{0} \cos t+p_{x_{0}} \sin t\right)^{4}}{8\left(p_{x_{0}} \cos t-x_{0} \sin t\right)^{2}} d t=-\frac{3}{8} \pi p_{x_{0}}\left(x_{0}^{2}+p_{x_{0}}^{2}\right),
$$


where $p_{x_{0}}=\sqrt{2 h-x_{0}^{2}}$ at the energy level $H=h>0$. Thus

$$
\mathcal{F}\left(x_{0}\right)=-\frac{3}{4} \pi h \sqrt{2 h-x_{0}^{2}} .
$$

The zeros of $\mathcal{F}\left(x_{0}\right)=0$ are $x_{0}= \pm \sqrt{2 h}$, which implies $p_{x_{0}}=0$. Since $\mathcal{F}^{\prime}( \pm \sqrt{2 h}) \neq$ 0 , both zeros of $\mathcal{F}\left(x_{0}\right)$ provide two initial conditions of the same periodic orbit for the perturbed differential system in the energy level $H=h>0$. Hence the proof of Theorem 1 for case $k=1$ follows.

Proof of Theorem 1 for $k=2$. We have the unperturbed periodic solution

$$
\begin{aligned}
x(t) & =0, \\
p_{x}(t) & =0, \\
y(t) & =y_{0} \cos \left(\frac{t}{q}\right)+p_{y_{0}} q \sin \left(\frac{t}{q}\right), \\
p_{y}(t) & =p_{y_{0}} \cos \left(\frac{t}{q}\right)-\frac{y_{0}}{q} \sin \left(\frac{t}{q}\right), \\
z(t) & =0, \\
p_{z}(t) & =0 .
\end{aligned}
$$

in the plane $\left(0, y, 0,0, p_{y}, 0\right)$. Note that these periodic solutions living in the plane $\left(0, y, 0,0, p_{y}, 0,0\right)$ are not in resonance with the other periodic solutions of system (7) for $\varepsilon=0$ living in the planes $\left(x, 0,0, p_{x}, 0,0\right)$ and $\left(0,0, z, 0,0, p_{z}\right)$ due to the fact that $q$ and $q^{1 / 2}$ are irrational. The first integral (8) when $\varepsilon=0$ takes on these periodic solutions the value

$$
h=\frac{1}{2}\left(p_{y_{0}}^{2}+\frac{y_{0}^{2}}{q^{2}}\right) .
$$

We apply the Averaging Theorem to every fixed energy level $H=h>0$. This allows to eliminate one of the coordinates, in this case $p_{y}$ and to reduce the study to dimension 5 . Then $p_{y}$ at the energy level $H=h$ with $h$ given by (11)

$$
\begin{aligned}
& p_{y}= \sqrt{p_{y_{0}}^{2}-p_{x}^{2}-\frac{p_{z}^{2}}{q}+\frac{y_{0}^{2}}{q^{2}}-x^{2}-\frac{y^{2}+z^{2}}{q^{2}}} \\
&+\varepsilon \frac{\left(q^{2} x^{2}+y^{2}+z^{2}\right)^{2}}{8 q^{4} \sqrt{p_{y_{0}}^{2}-p_{x}^{2}-\frac{p_{z}^{2}}{q}+\frac{y_{0}^{2}}{q^{2}}-x^{2}-\frac{y^{2}+z^{2}}{q^{2}}}}+\mathcal{O}\left(\varepsilon^{2}\right) .
\end{aligned}
$$


The equations of motion (7) on the energy level $H=h>0$ are given by

$$
\begin{aligned}
\dot{y}= & \sqrt{p_{y_{0}}^{2}-p_{x}^{2}-\frac{p_{z}^{2}}{q}+\frac{y_{0}^{2}}{q^{2}}-x^{2}-\frac{y^{2}+z^{2}}{q^{2}}} \\
& \varepsilon \frac{\left(q^{2} x^{2}+y^{2}+z^{2}\right)^{2}}{p^{4} \sqrt{p_{y_{0}}^{2}-p_{x}^{2}-\frac{p_{z}^{2}}{q}+\frac{y_{0}^{2}}{q^{2}}-x^{2}-d \frac{y^{2}+z^{2}}{q^{2}}}}+\mathcal{O}\left(\varepsilon^{2}\right), \\
\dot{x}= & p_{x}, \\
\dot{p}_{x}= & -x+\varepsilon \frac{x\left(q^{2} x^{2}+y^{2}+z^{2}\right)}{2 q^{2}}+\mathcal{O}\left(\varepsilon^{2}\right), \\
\dot{z}= & \frac{p_{z}}{q}, \\
\dot{p}_{z}= & -\frac{z}{q^{2}}+\varepsilon \frac{z\left(q^{2} x^{2}+y^{2}+z^{2}\right)}{2 q^{4}}+\mathcal{O}\left(\varepsilon^{2}\right) .
\end{aligned}
$$

Now this differential system has the form $\dot{\mathbf{x}}=F_{0}(t, \mathbf{x})+\varepsilon F_{1}(t, \mathbf{x})+\varepsilon^{2} F_{2}(t, \mathbf{x}, \varepsilon)$, where $\mathbf{x}=\left(y, x, p_{x}, z, p_{z}\right)$,

$$
F_{0}\left(y, x, p_{x}, z, p_{z}\right)=\left(\sqrt{p_{y_{0}}^{2}-p_{x}^{2}-\frac{p_{z}^{2}}{q}+\frac{y_{0}^{2}}{q^{2}}-x^{2}-\frac{y^{2}+z^{2}}{q^{2}}}, p_{x},-x, \frac{p_{z}}{q},-\frac{z}{q^{2}}\right)
$$

and $F_{1}\left(y, x, p_{x}, z, p_{z}\right)$ is

$$
\left(\frac{\left(q^{2} x^{2}+y^{2}+z^{2}\right)^{2}}{8 q^{4} \sqrt{p_{y_{0}}^{2}-p_{x}^{2}-\frac{p_{z}^{2}}{q}+\frac{y_{0}^{2}}{q^{2}}-x^{2}-\frac{y^{2}+z^{2}}{q^{2}}}}, 0, \frac{x\left(q^{2} x^{2}+y^{2}+z^{2}\right)}{2 q^{2}}, 0, \frac{z\left(q^{2} x^{2}+y^{2}+z^{2}\right)}{2 q^{4}}\right) .
$$

We have $k=1, n=5, \alpha=y_{0}, \mathbf{z}_{\alpha}=\left(y_{0}, 0,0,0,0\right), \beta_{0}: \mathbb{R} \longrightarrow \mathbb{R}^{4}$ is $\beta_{0}(\alpha)=$ $(0,0,0,0)$, is the initial condition of the unperturbed periodic orbits, for each $\mathbf{z}_{y_{0}}$ the solution $\mathbf{x}\left(t, \mathbf{z}_{y_{0}}\right)=\left(y_{0} \cos (t / q)+p_{y_{0}} q \sin (t / q), 0,0,0,0\right)$ is $2 \pi q$-periodic, and $\xi\left(y, x, p_{x}, z, p_{z}\right)=y$.

Let $\mathbf{M}_{\mathbf{z}_{y_{0}}}(t)$ be the fundamental matrix satisfying $\mathbf{M}_{\mathbf{z}_{y_{0}}}(0)=\mathbf{I}$ solution of the variational equation (22) along the periodic solutions $\mathbf{x}\left(t, \mathbf{z}_{y_{0}}\right)$. Then $\mathbf{M}_{\mathbf{z}_{y_{0}}}(t)$ is given by

$$
\left(\begin{array}{ccccc}
\cos \left(\frac{t}{q}\right)-\frac{y_{0}}{p_{y_{0}} q} \sin \left(\frac{t}{q}\right) & 0 & 0 & 0 & 0 \\
0 & \cos t & \sin t & 0 & 0 \\
0 & -\sin t & \cos t & 0 & 0 \\
0 & 0 & 0 & \cos \left(\frac{t}{q^{3 / 2}}\right) & q^{1 / 2} \sin \left(\frac{t}{q^{3 / 2}}\right) \\
0 & 0 & 0 & -\frac{1}{q^{1 / 2}} \sin \left(\frac{t}{q^{3 / 2}}\right) & \cos \left(\frac{t}{q^{3 / 2}}\right)
\end{array}\right) .
$$


Now we verify the condition $\operatorname{det} \Delta_{y_{0}} \neq 0$, then we compute $\mathbf{M}_{\mathbf{z}_{y_{0}}}^{-1}(0)-\mathbf{M}_{\mathbf{z}_{y_{0}}}^{-1}(2 \pi q)$ and we get

$$
\left(\begin{array}{ccccc}
0 & 0 & 0 & 0 & 0 \\
0 & 2 \sin ^{2}(\pi q) & \sin (2 \pi q) & 0 & 0 \\
0 & -\sin (2 \pi q) & 2 \sin ^{2}(\pi q) & 0 & 0 \\
0 & 0 & 0 & 2 \sin ^{2}\left(\frac{\pi}{q^{1 / 2}}\right) & q^{1 / 2} \sin \left(\frac{2 \pi}{q^{1 / 2}}\right) \\
0 & 0 & 0 & -\frac{1}{q^{1 / 2}} \sin \left(\frac{2 \pi}{q^{1 / 2}}\right) & 2 \sin ^{2}\left(\frac{\pi}{q^{1 / 2}}\right)
\end{array}\right)
$$

Therefore

$$
\operatorname{det} \Delta_{y_{0}}=16 \sin ^{2}\left(\frac{\pi}{q^{1 / 2}}\right) \sin ^{2}(\pi q) \neq 0
$$

The function $F_{1}$ along the periodic orbit is given by

$$
F_{1}\left(t, \mathbf{x}\left(t, \mathbf{z}_{y_{0}}\right)\right)=\left(\frac{p_{y_{0}}\left(y_{0} \cos \left(\frac{t}{q}\right)+p_{y_{0}} q \sin \left(\frac{t}{q}\right)\right)^{4}}{8 q^{3}\left(p_{y_{0}} q \cos \left(\frac{t}{q}\right)-y_{0} \sin \left(\frac{t}{q}\right)\right)}, 0,0,0,0\right)
$$

then

$$
\mathbf{M}_{\mathbf{z}_{y_{0}}}^{-1}(t) F_{1}\left(t, \mathbf{x}\left(t, \mathbf{z}_{y_{0}}\right)\right)=\left(\frac{p_{y_{0}}\left(y_{0} \cos \left(\frac{t}{q}\right)+p_{y_{0}} q \sin \left(\frac{t}{q}\right)\right)^{4}}{8 q^{2}\left(p_{y_{0}} q \cos \left(\frac{t}{q}\right)-y_{0} \sin \left(\frac{t}{q}\right)\right)^{2}}, 0,0,0,0\right)
$$

From (23) we have

$$
\mathcal{F}\left(y_{0}\right)=\int_{0}^{2 \pi q} \frac{p_{y_{0}}\left(y_{0} \cos \left(\frac{t}{q}\right)+p_{y_{0}} q \sin \left(\frac{t}{q}\right)\right)^{4}}{8 q^{2}\left(p_{y_{0}} q \cos \left(\frac{t}{q}\right)-y_{0} \sin \left(\frac{t}{q}\right)\right)^{2}} d t=-\frac{3}{8 q} \pi p_{y_{0}}\left(y_{0}^{2}+p_{y_{0}}^{2} q^{2}\right)
$$

where $p_{y_{0}}=\sqrt{2 h-y_{0}^{2} / q^{2}}$ at the energy level. Thus

$$
\mathcal{F}\left(y_{0}\right)=-\frac{3}{4} \pi h \sqrt{2 h q^{2}-y_{0}^{2}}
$$

The zeros of $\mathcal{F}\left(y_{0}\right)=0$ are $y_{0}= \pm 2 \sqrt{h} q$, which implies $p_{y_{0}}=0$. Since $\mathcal{F}^{\prime}( \pm 2 \sqrt{h} q) \neq 0$, both zeros provide two initial conditions of the same periodic orbit for the perturbed differential system in the energy level $H=h>0$. So the proof of Theorem 1 for case $k=2$ is done. 
Proof of Theorem 1 for $k=3$. Now we consider, the unperturbed periodic solutions

$$
\begin{aligned}
x(t) & =0 \\
p_{x}(t) & =0, \\
y(t) & =0 \\
p_{y}(t) & =0, \\
z(t) & =z_{0} \cos \left(\frac{t}{q^{3 / 2}}\right)+p_{z_{0}} q^{1 / 2} \sin \left(\frac{t}{q^{3 / 2}}\right), \\
p_{z}(t) & =p_{z_{0}} \cos \left(\frac{t}{q^{3 / 2}}\right)-\frac{z_{0}}{q^{1 / 2}} \sin \left(\frac{t}{q^{3 / 2}}\right) .
\end{aligned}
$$

in the plane $\left(0,0, z, 0,0, p_{z}\right)$. Note that these periodic solutions living in the plane $\left(0,0, z, 0,0, p_{z}\right)$ are not in resonance with the other periodic solutions of system (7) for $\varepsilon=0$ living in the planes $\left(x, 0,0, p_{x}, 0,0\right)$ and $\left(0, y, 0,0, p_{y}, 0,0\right)$ due to the fact that $q^{1 / 2}$ and $q^{3 / 2}$ are irrational. The first integral (8) when $\varepsilon=0$ takes on these periodic solutions the value $h=\left(p_{z_{0}}^{2} q+z_{0}^{2}\right) /\left(2 q^{2}\right)$.

We apply the Averaging Theorem, to every fixed energy level, $H=h>0$. This allows to eliminate one of the coordinates, in this case $p_{z}$. Then $p_{z}$ at the energy level $H=h$ is

$$
\begin{aligned}
p_{z}= & \sqrt{p_{z_{0}}^{2}-q\left(p_{x}^{2}+p_{y}^{2}\right)+\frac{z_{0}^{2}}{q}-q x^{2}-\frac{y^{2}+z^{2}}{q}} \\
& +\varepsilon \frac{\left(q^{2} x^{2}+y^{2}+z^{2}\right)^{2}}{8 q^{3} \sqrt{p_{z_{0}}^{2}-q\left(p_{x}^{2}+p_{y}^{2}\right)+\frac{z_{0}^{2}}{q}-q x^{2}-\frac{y^{2}+z^{2}}{q}}}+\mathcal{O}\left(\varepsilon^{2}\right) .
\end{aligned}
$$

The equations of motion (7) on the energy level $H=h>0$ are given by

$$
\begin{aligned}
\dot{z}= & \sqrt{p_{z_{0}}^{2}-q\left(p_{x}^{2}+p_{y}^{2}\right)+\frac{z_{0}^{2}}{q}-q x^{2}-\frac{y^{2}+z^{2}}{q}} \\
& +\varepsilon \frac{\left(q^{2} x^{2}+y^{2}+z^{2}\right)^{2}}{8 q^{3} \sqrt{p_{z_{0}}^{2}-q\left(p_{x}^{2}+p_{y}^{2}\right)+\frac{z_{0}^{2}}{q}-q x^{2}-\frac{y^{2}+z^{2}}{q}}}+\mathcal{O}\left(\varepsilon^{2}\right), \\
\dot{x}= & p_{x}, \\
\dot{p}_{x}= & -x+\varepsilon \frac{x\left(q^{2} x^{2}+y^{2}+z^{2}\right)}{2 q^{2}}+\mathcal{O}\left(\varepsilon^{2}\right), \\
\dot{y}= & p_{y}, \\
\dot{p}_{y}= & -\frac{y}{q^{2}}+\varepsilon \frac{y\left(q^{2} x^{2}+y^{2}+z^{2}\right)}{2 q^{4}}+\mathcal{O}\left(\varepsilon^{2}\right) .
\end{aligned}
$$


Now this differential system has the form $\dot{\mathbf{x}}=F_{0}(t, \mathbf{x})+\varepsilon F_{1}(t, \mathbf{x})+\varepsilon^{2} F_{2}(t, \mathbf{x}, \varepsilon)$, where $\mathbf{x}=\left(z, x, p_{x}, y, p_{y}\right)$,

$$
F_{0}\left(z, x, p_{x}, y, p_{y}=\left(\sqrt{p_{z_{0}}^{2}-q\left(p_{x}^{2}+p_{y}^{2}\right)+\frac{z_{0}^{2}}{q}-q x^{2}-\frac{y^{2}+z^{2}}{q}}, p_{x},-x, p_{y},-\frac{y}{q^{2}}\right)\right.
$$

and $F_{1}\left(z, x, p_{x}, y, p_{y}\right)$ is

$$
\left(\frac{\left(q^{2} x^{2}+y^{2}+z^{2}\right)^{2}}{8 q^{4} \sqrt{p_{x_{0}}^{2}-p_{y}^{2} \frac{p_{z}^{2}}{q}+x_{0}^{2}-x^{2}-\frac{y^{2}+z^{2}}{q^{2}}}}, 0, \frac{y\left(q^{2} x^{2}+y^{2}+z^{2}\right)}{2 q^{4}}, 0, \frac{z\left(q^{2} x^{2}+y^{2}+z^{2}\right)}{2 q^{4}}\right) .
$$

We have $k=1, n=5, \alpha=z_{0}, \beta_{0}: \mathbb{R} \longrightarrow \mathbb{R}^{4}$ is $\beta_{0}(\alpha)=(0,0,0,0), \mathbf{z}_{\alpha}=$ $\left(z_{0}, 0,0,0,0\right)$ is the initial condition of the unperturbed periodic orbits, and for each $\mathbf{z}_{z_{0}}$, the solution $\mathbf{x}\left(t, \mathbf{z}_{z_{0}}\right)=\left(z_{0} \cos \left(t / q^{3 / 2}\right)+p_{z_{0}} q^{1 / 2} \sin \left(t / q^{3 / 2}\right), 0,0,0,0\right)$ is $2 \pi q^{3 / 2}$-periodic, and $\xi\left(z, x, p_{x}, y, p_{y}\right)=z$.

Let $\mathbf{M}_{\mathbf{z}_{z_{0}}}(t)$ be the fundamental matrix satisfying $\mathbf{M}_{\mathbf{z}_{z_{0}}}(0)=\mathbf{I}$ solution of the variational equation (22) along the periodic solutions $\mathbf{x}\left(t, \mathbf{z}_{z_{0}}\right)$. Then $\mathbf{M}_{\mathbf{z}_{z_{0}}}(t)$ is given by

$$
\left(\begin{array}{ccccc}
\cos \left(\frac{t}{q^{3 / 2}}\right)-\frac{z_{0}}{p_{z_{0}} q^{1 / 2}} \sin \left(\frac{t}{q^{3 / 2}}\right) & 0 & 0 & 0 & 0 \\
0 & \cos t & \sin t & 0 & 0 \\
0 & -\sin t & \cos t & 0 & 0 \\
0 & 0 & 0 & \cos \left(\frac{t}{q}\right) & q \sin \left(\frac{t}{q}\right) \\
0 & 0 & 0 & -\frac{1}{q} \sin \left(\frac{t}{q}\right) & \cos \left(\frac{t}{q}\right)
\end{array}\right)
$$

We have $\mathbf{M}_{\mathbf{z}_{z_{0}}}^{-1}(0)-\mathbf{M}_{\mathbf{z}_{z_{0}}}^{-1}\left(2 \pi q^{3 / 2}\right)$ equal to

$$
\left(\begin{array}{ccccc}
0 & 0 & 0 & 0 & 0 \\
0 & 2 \sin ^{2}\left(\pi q^{3 / 2}\right) & q \sin \left(2 \pi q^{3 / 2}\right) & 0 & 0 \\
0 & -\sin \left(2 \pi q^{3 / 2}\right) & 2 \sin ^{2}\left(\pi q^{3 / 2}\right) & 0 & 0 \\
0 & 0 & 0 & 2 \sin ^{2}\left(\pi q^{1 / 2}\right) & q \sin \left(2 \pi q^{1 / 2}\right) \\
0 & 0 & 0 & -\frac{\sin \left(2 \pi q^{1 / 2}\right)}{q} & 2 \sin ^{2}\left(\pi q^{1 / 2}\right)
\end{array}\right)
$$

Then

$$
\operatorname{det} \Delta_{z_{0}}=16 \sin ^{2}\left(\pi q^{1 / 2}\right) \sin ^{2}\left(\pi q^{3 / 2}\right) \neq 0 .
$$

The function $F_{1}$ along the periodic orbit is given by

$$
F_{1}\left(t, \mathbf{x}\left(t, \mathbf{z}_{z_{0}}\right)\right)=\left(\frac{\left(z_{0} \cos \left(\frac{t}{q^{3 / 2}}\right)+p_{z_{0}} q^{1 / 2} \sin \left(\frac{t}{q^{3 / 2}}\right)\right)^{4}}{8 q^{7 / 2}\left(p_{z_{0}} q^{1 / 2} \cos \left(\frac{t}{q^{3 / 2}}\right)-z_{0} \sin \left(\frac{t}{q^{3 / 2}}\right)\right)}, 0,0,0,0\right)
$$


then $\mathbf{M}_{\mathbf{z}_{z_{0}}}^{-1}(t) F_{1}\left(t, \mathbf{x}\left(t, \mathbf{z}_{z_{0}}\right)\right)$ is given by

$$
\left(\frac{\left(z_{0} \cos \left(\frac{t}{q^{3 / 2}}\right)+p_{z_{0}} q^{1 / 2} \sin \left(\frac{t}{q^{3 / 2}}\right)\right)^{4}}{8 q^{7 / 2}\left(p_{z_{0}} q^{1 / 2} \cos \left(\frac{t}{q^{3 / 2}}\right)-z_{0} \sin \left(\frac{t}{q^{3 / 2}}\right)\right)\left(\cos \left(\frac{t}{q^{3 / 2}}\right)-\frac{z_{0}}{p_{z_{0}} q^{1 / 2}} \sin \left(\frac{t}{q^{3 / 2}}\right)\right)}, 0,0,0,0\right) .
$$

Therefore, from (23), the function $\mathcal{F}\left(z_{0}\right)$ is

$$
\begin{aligned}
& \int_{0}^{2 \pi q^{3 / 2}} \frac{\left(z_{0} \cos \left(\frac{t}{q^{3 / 2}}\right)+p_{z_{0}} q^{1 / 2} \sin \left(\frac{t}{q^{3 / 2}}\right)\right)^{4}}{8 q^{7 / 2}\left(p_{z_{0}} q^{1 / 2} \cos \left(\frac{t}{q^{3 / 2}}\right)-z_{0} \sin \left(\frac{t}{q^{3 / 2}}\right)\right)\left(\cos \left(\frac{t}{q^{3 / 2}}\right)-\frac{1}{q^{1 / 2}} \frac{z_{0}}{p_{z_{0}}} \sin \left(\frac{t}{q^{3 / 2}}\right)\right)} d t \\
& =-\frac{3 \pi}{8 q^{3 / 2}} p_{z_{0}}\left(z_{0}^{2}+p_{z_{0}}^{2} q\right),
\end{aligned}
$$

where $p_{z_{0}}=\sqrt{\left(2 h q^{2}-z_{0}^{2}\right) / q}$ at the energy level. Thus

$$
\mathcal{F}\left(z_{0}\right)=-\frac{3 \pi}{4} h \sqrt{2 h q^{2}-z_{0}^{2}} .
$$

The zeros of $\mathcal{F}\left(z_{0}\right)=0$ are $z_{0}= \pm \sqrt{2 h} q$, which implies $p_{z_{0}}=0$. Since $\mathcal{F}^{\prime}( \pm \sqrt{2 h} q) \neq 0$, both zeros of $\mathcal{F}\left(z_{0}\right)$ provide initial conditions of the same periodic orbit for the perturbed differential system in the energy level $H=h>0$. This completes the proof of Theorem 1 for case $k=3$.

\section{Proof of Theorem 2}

We consider the case $q$ is rational and $q^{3 / 2}$ is irrational and we shall obtain two families of periodic orbits.

Proof of Theorem 2 for $k=1$. We consider the unperturbed periodic solutions

$$
\begin{aligned}
x(t) & =x_{0} \cos t+p_{x_{0}} \sin t \\
p_{x}(t) & =p_{x_{0}} \cos t-x_{0} \sin t \\
y(t) & =y_{0} \cos \left(\frac{t}{q}\right)+p_{y_{0}} q \sin \left(\frac{t}{q}\right), \\
p_{y}(t) & =p_{y_{0}} \cos \left(\frac{t}{q}\right)-\frac{y_{0}}{q} \sin \left(\frac{t}{q}\right), \\
z(t) & =0 \\
p_{z}(t) & =0 .
\end{aligned}
$$

in the subspace $\left(x, y, 0, p_{x}, p_{y}, 0\right)$. Note that these periodic solutions living in the space $\left(x, y, 0, p_{x}, p_{y}, 0\right)$ are not in resonance with the periodic solutions of system (7) for $\varepsilon=0$ living in the plane $\left(0,0, z, 0,0, p_{z}\right)$ because $q$ is rational and $q^{3 / 2}$ are irrational. The first integral ( 8 ) when $\varepsilon=0$ takes on these periodic solutions the value

$$
h=\frac{1}{2}\left(p_{x_{0}}^{2}+p_{y_{0}}^{2}+x_{0}^{2}+\frac{y_{0}^{2}}{q^{2}}\right) .
$$

We apply the Averaging Theorem to every fixed energy level $H=h>0$. This allows to eliminate one of the coordinates, in this case $p_{x}$ and to reduce the study 
to dimension 5. At the energy level $H=h$ with $h$ given by (16) we have that

$$
\begin{aligned}
p_{x}= & \sqrt{p_{x_{0}}^{2}+p_{y_{0}}^{2}-p_{y}^{2}-\frac{p_{z}^{2}}{q}+x_{0}^{2}+\frac{y_{0}^{2}}{q^{2}}-x^{2}-\frac{y^{2}+z^{2}}{q^{2}}} \\
+ & \varepsilon \frac{\left(q^{2} x^{2}+y^{2}+z^{2}\right)^{2}}{8 q^{4} \sqrt{p_{x_{0}}^{2}+p_{y_{0}}^{2}-p_{y}^{2}-\frac{p_{z}^{2}}{q}+x_{0}^{2}+\frac{y_{0}^{2}}{q^{2}}-x^{2}-\frac{y^{2}+z^{2}}{q^{2}}}}+\mathcal{O}\left(\varepsilon^{2}\right) .
\end{aligned}
$$

The equations of motion (7) on the energy level $H=h$ are given by

$$
\begin{aligned}
\dot{x}= & \sqrt{p_{x_{0}}^{2}+p_{y_{0}}^{2}-p_{y}^{2}-\frac{p_{z}^{2}}{q}+x_{0}^{2}+\frac{y_{0}^{2}}{q^{2}}-x^{2}-\frac{y^{2}+z^{2}}{q^{2}}} \\
& +\varepsilon \frac{\left(q^{2} x^{2}+y^{2}+z^{2}\right)^{2}}{8 q^{4} \sqrt{p_{x_{0}}^{2}+p_{y_{0}}^{2}-p_{y}^{2}-\frac{p_{z}^{2}}{q}+x_{0}^{2}+\frac{y_{0}^{2}}{q^{2}}-x^{2}-\frac{y^{2}+z^{2}}{q^{2}}}+\mathcal{O}\left(\varepsilon^{2}\right) .} \\
(17) \quad \dot{y}_{y}= & p_{y} \\
\dot{p}_{y}= & -\frac{y}{q^{2}}+\varepsilon \frac{y\left(q^{2} x^{2}+y^{2}+z^{2}\right)}{2 q^{4}}+\mathcal{O}\left(\varepsilon^{2}\right), \\
\dot{z}= & \frac{p_{z}}{q}, \\
\dot{p}_{z}= & -\frac{z}{q^{2}}+\varepsilon \frac{z\left(q^{2} x^{2}+y^{2}+z^{2}\right)}{2 q^{4}}+\mathcal{O}\left(\varepsilon^{2}\right) .
\end{aligned}
$$

Now this differential system has the form $\dot{\mathbf{x}}=F_{0}(t, \mathbf{x})+\varepsilon F_{1}(t, \mathbf{x})+\varepsilon^{2} F_{2}(t, \mathbf{x}, \varepsilon)$, where $\mathbf{x}=\left(x, y, p_{y}, z, p_{z}\right)$ and $F_{0}\left(x, y, p_{y}, z, p_{z}\right)$ is

$$
\left(\sqrt{p_{x_{0}}^{2}+p_{y_{0}}^{2}-p_{y}^{2}-\frac{p_{z}^{2}}{q}+x_{0}^{2}+\frac{y_{0}^{2}}{q^{2}}-x^{2}-\frac{y^{2}+z^{2}}{q^{2}}}, p_{y},-\frac{y}{q^{2}}, \frac{p_{z}}{q},-\frac{z}{q^{2}}\right) .
$$

The function

$$
F_{1}\left(x, y, p_{y}, z, p_{z}\right)=\left(A, 0, \frac{y\left(q^{2} x^{2}+y^{2}+z^{2}\right)}{2 q^{4}}, 0, \frac{z\left(q^{2} x^{2}+y^{2}+z^{2}\right)}{2 q^{4}}\right),
$$

with

$$
A=\frac{\left(q^{2} x^{2}+y^{2}+z^{2}\right)^{2}}{8 q^{4} \sqrt{p_{x_{0}}^{2}+p_{y_{0}}^{2}-p_{y}^{2}-\frac{p_{z}^{2}}{q}+x_{0}^{2}+\frac{y_{0}^{2}}{q^{2}}-x^{2}-\frac{y^{2}+z^{2}}{q^{2}}}} .
$$

We have $k=3, n=5, \alpha=\left(x_{0}, y_{0}, p_{y_{0}}\right), \beta_{0}: \mathbb{R}^{3} \longrightarrow \mathbb{R}^{2}$ is $\beta_{0}(\alpha)=(0,0), \mathbf{z}_{\alpha}=$ $\left(x_{0}, y_{0}, p_{y_{0}}, 0,0\right)$ is the initial condition of the unperturbed periodic orbits, and for each $\mathbf{z}_{\alpha}$, the solution $\mathbf{x}\left(t, \mathbf{z}_{\alpha}\right)=\left(x_{0} \cos t+p_{x_{0}} \sin t, y_{0} \cos (t / q)+p_{y_{0}} q \sin (t / q), p_{y_{0}} \cos (t / q)-\right.$ $\left.\left.y_{0} / q \sin (t / q), 0,0\right)\right)$ is $2 \pi m$-periodic, where $q=m / n$ with $(m, n)=1$ and $\xi\left(x, y, p_{y}, z, p_{z}\right)=$ $\left(x, y, p_{y}\right)$.

The fundamental matrix $\mathbf{M}_{\mathbf{z}_{\alpha}}(t)$ satisfying $\mathbf{M}_{\mathbf{z}_{\alpha}}(0)=\mathbf{I}$ is the solution of the variational equation (22) along the periodic solutions $\mathbf{x}\left(t, \mathbf{z}_{\alpha}\right)$. Then $\mathbf{M}_{\mathbf{z}_{\alpha}}(t)$ is given 
by

$$
\left(\begin{array}{ccccc}
\cos t-\frac{x_{0} \sin t}{p_{x_{0}}} & -\frac{y_{0} \sin t}{p_{x_{0}} q^{2}} & -\frac{p_{y_{0}} \sin t}{p_{x_{0}}} & 0 & 0 \\
0 & \cos \left(\frac{t}{q}\right) & q \sin \left(\frac{t}{q}\right) & 0 & 0 \\
0 & -\frac{1}{q} \sin \left(\frac{t}{q}\right) & \cos \left(\frac{t}{q}\right) & 0 & 0 \\
0 & 0 & 0 & \cos \left(\frac{t}{q^{3 / 2}}\right) & q^{1 / 2} \sin \left(\frac{t}{q^{3 / 2}}\right) \\
0 & 0 & 0 & -\frac{1}{q^{1 / 2}} \sin \left(\frac{t}{q^{3 / 2}}\right) & \cos \left(\frac{t}{q^{3 / 2}}\right)
\end{array}\right)
$$

With the calculus of $\mathbf{M}_{\mathbf{z}_{\alpha}}^{-1}(0)-\mathbf{M}_{\mathbf{z}_{\alpha}}^{-1}(2 \pi m)$, we verify that

$$
\operatorname{det} \Delta_{z_{\alpha}}=4 \sin ^{2}\left(\frac{\pi n^{3 / 2}}{n^{1 / 2}}\right) \neq 0
$$

The function $F_{1}$ along the periodic orbit is given by $F_{1}\left(t, \mathbf{x}\left(t, \mathbf{z}_{\alpha}\right)\right)=(B, 0, C, 0,0)$, where

$$
\begin{aligned}
B= & \frac{1}{8 m^{4}\left(p_{x_{0}} \cos t-x_{0} \sin t\right)}\left[n^{2} y_{0}^{2} \cos ^{2}\left(\frac{n t}{m}\right)\right. \\
& \left.+m\left(m\left(\left(x_{0} \cos t+p_{x_{0}} \sin t\right)^{2}+p_{y_{0}}^{2} \sin \left(\frac{n t}{m}\right)^{2}\right)+n p_{y_{0}} y_{0} \sin \left(\frac{2 n t}{m}\right)\right)\right]^{2}, \\
C= & \frac{n^{4}}{2 m^{4}}\left(y_{0} \cos \left(\frac{n t}{m}\right)+\frac{m}{n} p_{y_{0}} \sin \left(\frac{n t}{m}\right)\right) \\
& \left(\frac{m^{2}}{n^{2}}\left(x_{0} \cos t+p_{x_{0}} \sin t\right)^{2}+\left(y_{0} \cos \left(\frac{n t}{m}\right)+\frac{m}{n} p_{y_{0}} \sin \left(\frac{n t}{m}\right)\right)^{2}\right) .
\end{aligned}
$$

Then $\mathbf{M}_{\mathbf{z}_{\alpha}}^{-1}(t) F_{1}\left(t, \mathbf{x}\left(t, \mathbf{z}_{\alpha}\right)\right)=(D, E, F, 0,0)$, where

$$
\begin{aligned}
D= & \frac{n^{5}}{2 m^{5}\left(p_{x_{0}} \cos t-x_{0} \sin t\right)}\left(y_{0} \cos \left(\frac{n t}{m}\right)+\frac{m}{n} p_{y_{0}} \sin \left(\frac{n t}{m}\right)\right) \\
& \left(\frac{m}{n} p_{y_{0}} \cos \left(\frac{n t}{m}\right) \sin t-y_{0} \sin t \sin \left(\frac{n t}{m}\right)\right) \\
& \left(\frac{m^{2}}{n^{2}}\left(x_{0} \cos t+p_{x_{0}} \sin t\right)^{2}+\left(y_{0} \cos \left(\frac{n t}{m}\right)+\frac{m}{n} p_{y_{0}} \sin \left(\frac{n t}{m}\right)\right)^{2}\right) \\
& +\frac{1}{8 m^{4}\left(p_{x_{0}} \cos t-x_{0} \sin t\right)^{2}}\left\{p _ { x _ { 0 } } \left[n^{2} y_{0}^{2} \cos ^{2}\left(\frac{n t}{m}\right)\right.\right. \\
& \left.\left.+m\left(m\left(\left(x_{0} \cos t+p_{x_{0}} \sin t\right)^{2}+p_{y_{0}}^{2} \sin ^{2}\left(\frac{n t}{m}\right)\right)+n p_{y_{0}} y_{0} \sin \left(\frac{2 n t}{m}\right)\right)\right]^{2}\right\},
\end{aligned}
$$




$$
\begin{aligned}
E= & -\frac{n^{3}}{2 m^{3}} \sin \left(\frac{n t}{m}\right)\left(y_{0} \cos \left(\frac{n t}{m}\right)+\frac{m}{n} p_{y_{0}} \sin \left(\frac{n t}{m}\right)\right) \\
& \left(\frac{m^{2}}{n^{2}}\left(x_{0} \cos t+p_{x_{0}} \sin t\right)^{2}+\left(y_{0} \cos \left(\frac{n t}{m}\right)+\frac{m}{n} p_{y_{0}} \sin \left(\frac{n t}{m}\right)\right)^{2}\right) \\
F= & \frac{n^{4}}{2 m^{4}} \cos \left(\frac{n t}{m}\right)\left(y_{0} \cos \left(\frac{n t}{m}\right)+\frac{m}{n} p_{y_{0}} \sin \left(\frac{n t}{m}\right)\right) \\
& \left(\frac{m^{2}}{n^{2}}\left(x_{0} \cos t+p_{x_{0}} \sin t\right)^{2}+\left(y_{0} \cos \left(\frac{n t}{m}\right)+\frac{m}{n} p_{y_{0}} \sin \left(\frac{n t}{m}\right)\right)^{2}\right)
\end{aligned}
$$

The function $\mathcal{F}(\alpha)=\left(\mathcal{F}_{D}, \mathcal{F}_{E}, \mathcal{F}_{F}\right)$ where $\mathcal{F}_{D}, \mathcal{F}_{E}, \mathcal{F}_{F}$ are the projections in the first, second and third component, respectively, of the integral of $\mathbf{M}_{\mathbf{z}_{\alpha}}^{-1}(t) F_{1}\left(t, \mathbf{x}\left(t, \mathbf{z}_{\alpha}\right)\right)$ in one period, i. e.,

$$
\begin{aligned}
& \mathcal{F}_{D}(\alpha)=\int_{0}^{2 \pi m} D d t=-\frac{\pi}{8 m} p_{x_{0}}\left(m^{2}\left(3 p_{x_{0}}^{2}+2 p_{y_{0}}^{2}+3 x_{0}^{2}\right)+2 n^{2} y_{0}^{2}\right), \\
& \mathcal{F}_{E}(\alpha)=\int_{0}^{2 \pi m} E d t=-\frac{\pi}{8 m} p_{y_{0}}\left(m^{2}\left(2 p_{x_{0}}^{2}+3 p_{y_{0}}^{2}+2 x_{0}^{2}\right)+3 n^{2} y_{0}^{2}\right), \\
& \mathcal{F}_{F}(\alpha)=\int_{0}^{2 \pi m} F d t=\frac{n^{2} \pi}{8 m^{3}} y_{0}\left(m^{2}\left(2 p_{x_{0}}^{2}+3 p_{y_{0}}^{2}+2 x_{0}^{2}\right)+3 n^{2} y_{0}^{2}\right),
\end{aligned}
$$

where $p_{x_{0}}=\sqrt{2 h-x_{0}^{2}-p_{y_{0}}^{2}-\left(n^{2} / m^{2}\right) y_{0}^{2}}$ at the energy level $H=h>0$. Thus

$$
\begin{aligned}
\mathcal{F}_{D}(\alpha) & =\frac{\pi}{8 m}\left(m^{2}\left(-6 h+p_{y_{0}}^{2}\right)+n^{2} y_{0}^{2}\right) \sqrt{2 h-p_{y_{0}}^{2}-x_{0}^{2}-\frac{n^{2}}{m^{2}} y_{0}^{2}}, \\
\mathcal{F}_{E}(\alpha) & =-\frac{\pi}{8 m} p_{y_{0}}\left(m^{2}\left(4 h+p_{y_{0}}^{2}\right)+n^{2} y_{0}^{2}\right) \\
\mathcal{F}_{F}(\alpha) & =\frac{\pi n^{2}}{8 m^{3}} y_{0}\left(m^{2}\left(4 h+p_{y_{0}}^{2}\right)+n^{2} y_{0}^{2}\right)
\end{aligned}
$$

The zeros of $\mathcal{F}(\alpha)=\mathbf{0}$ are $\left(x_{0}, y_{0}, p_{y_{0}}\right)=( \pm \sqrt{2 h}, 0,0)$, which implies $p_{x_{0}}=0$. Since

$$
\operatorname{det}\left(\left.\frac{\partial\left(\mathcal{F}_{D}, \mathcal{F}_{E}, \mathcal{F}_{F}\right)}{\partial\left(x_{0}, y_{0}, p_{y_{0}}\right)}\right|_{\left(x_{0}, y_{0}, p_{y_{0}}\right)=( \pm \sqrt{2 h}, 0,0)}\right) \neq 0
$$

both zeros provide initial conditions of the same periodic orbit for the perturbed differential system in the energy level $H=h>0$. This completes the proof of Theorem 2 for $k=1$. 
Proof of Theorem 2 for $k=2$. We consider the unperturbed periodic solution

$$
\begin{aligned}
x(t) & =0, \\
p_{x}(t) & =0, \\
y(t) & =0, \\
p_{y}(t) & =0, \\
z(t) & =z_{0} \cos \left(\frac{t}{q^{3 / 2}}\right)+p_{z_{0}} q^{1 / 2} \sin \left(\frac{t}{q^{3 / 2}}\right), \\
p_{z}(t) & =p_{z_{0}} \cos \left(\frac{t}{q^{3 / 2}}\right)-\frac{z_{0}}{q^{1 / 2}} \sin \left(\frac{t}{q^{3 / 2}}\right) .
\end{aligned}
$$

in the plane $(0,0, z, 0,0, p z)$. Note that these periodic solutions living in the plane $\left(0,0, z, 0,0, p_{z}\right)$ are not in resonance with the periodic solutions of the system (7) living in the space $\left(x, y, 0, p_{x}, p_{y}, 0\right)$ because $q$ is rational and $q^{3 / 2}$ is irrational.

The rest of the proof is analogous to the proof of case $k=3$ of Theorem 1 .

\section{Proof of Theorem 3}

We consider the case $q$ is irrational and $q^{3 / 2}$ is rational and we obtain only one family of periodic orbits.

Proof of Theorem 3. In this case, the unperturbed periodic solutions

$$
\begin{aligned}
x(t) & =0 \\
p_{x}(t) & =0 \\
y(t) & =y_{0} \cos \left(\frac{t}{q}\right)+p_{y_{0}} q \sin \left(\frac{t}{q}\right) \\
p_{y}(t) & =p_{y_{0}} \cos \left(\frac{t}{q}\right)-\frac{y_{0}}{q} \sin \left(\frac{t}{q}\right) \\
z(t) & =0 \\
p_{z}(t) & =0
\end{aligned}
$$

in the plane $\left(0, y, 0,0, p_{y}, 0\right)$. Note that these periodic solutions living in the plane $\left(0, y, 0,0, p_{y}, 0\right)$ are not in resonance with the periodic solutions of system (7) living in the space $\left(x, 0, z, p_{x}, 0, p_{z}\right)$ because $q$ is irrational and $q^{3 / 2}$ is rational.

From now the proof is analogous to the proof of case $k=2$ of Theorem 1 .

\section{Appendix: Averaging Theory}

Consider the differential equation

$$
\dot{\mathbf{x}}=F_{0}(t, \mathbf{x})+\varepsilon F_{1}(t, \mathbf{x})+\varepsilon F_{2}(t, \mathbf{x}, \varepsilon),
$$

with $\varepsilon>0$ sufficiently small, $\mathbf{x} \in \Omega$, where $\Omega$ is an open subset of $\mathbb{R}^{n}$, and $t \geq 0$. Moreover, we assume that both $F_{0}, F_{1}$ and $F_{2}$ are $\mathcal{C}^{2}$ functions and $T$-periodic in the first variable.

Let $\mathbf{x}(t, \mathbf{z})$ be the solution of the unperturbed system

$$
\dot{\mathbf{x}}=F_{0}(t, \mathbf{x}),
$$

such that $\mathbf{x}(0, \mathbf{z})=\mathbf{z}$. 
We write the linearization of the unperturbed system along the periodic solution $\mathbf{x}(t, \mathbf{z})$ as

$$
\dot{\mathbf{y}}(t)=D_{\mathbf{x}} F_{0}(t, \mathbf{x}(t, \mathbf{z})) \mathbf{y} .
$$

Now we denote by $M_{\mathbf{z}}(t)$ some fundamental matrix of the linear differential system $(22)$, and by $\xi: \mathbb{R}^{k} \times \mathbb{R}^{n-k} \longrightarrow \mathbb{R}^{k}$, the projection of $\mathbb{R}^{n}$ onto its first $k$ coordinates; i.e., $\xi\left(x_{1}, \ldots, x_{n}\right)=\left(x_{1}, \ldots, x_{k}\right)$.

Theorem 4 (First Order Averaging Theorem). Let $V \subset \mathbb{R}^{k}$ be open and bounded, and let $\beta_{0}: C L(V) \longrightarrow \mathbb{R}^{n-k}$ be a $\mathcal{C}^{2}$ function. We assume that

(i) $\mathcal{Z}=\left\{\mathbf{z}_{\alpha}=\left(\alpha, \beta_{0}(\alpha)\right), \alpha \in C l(V)\right\} \subset \Omega$ and that for each $\mathbf{z}_{\alpha} \in \mathcal{Z}$ the solution $\mathbf{x}\left(t, \mathbf{z}_{\alpha}\right)$ of $(21)$ is $T$-periodic;

(ii) for each $\mathbf{z}_{\alpha} \in \mathcal{Z}$ there is a fundamental matrix $M_{\mathbf{z}_{\alpha}}(t)$ of (22) such that the matrix $M_{\mathbf{z}_{\alpha}}^{-1}(0)-M_{\mathbf{z}_{\alpha}}^{-1}(T)$ has in the upper right corner the $k \times(n-k)$ zero matrix, and in the lower right corner $a(n-k) \times(n-k)$ matrix $\Delta_{\alpha}$ with det $\Delta_{\alpha} \neq 0$.

We consider the function $\mathcal{F}(\alpha): C l(V) \rightarrow \mathbb{R}^{k}$

$$
\mathcal{F}(\alpha)=\xi\left(\int_{0}^{T} M_{\mathbf{z}_{\alpha}}^{-1}(t) F_{1}\left(t, \mathbf{x}\left(t, \mathbf{z}_{\alpha}\right)\right) d t\right) .
$$

If there exists $a \in V$ with $\mathcal{F}(a)=0$ and

$$
\operatorname{det}((d \mathcal{F} / d \alpha(a)) \neq 0,
$$

then there is a $T$ - periodic solution $\phi(t, \epsilon)$ of system 20 such that $\phi(0, \epsilon) \rightarrow \mathbf{z}_{\alpha}$ as $\epsilon \rightarrow 0$.

For an easy proof of Theorem 4 see Corollary 1 of [2]. In fact the result of Theorem 4 is a classical result due to Malkin [5] and Roseau [9]

\section{ACKNOWLEDGEMENTS}

The first author is partially supported by CNPq grant 201802/2012-0. The second author is partially supported by a MINECO/FEDER grant MTM200803437, a CIRIT grant number 2009SGR-410, an ICREA Academia, and two grants FP7-PEOPLE-2012-IRSES 316338 and 318999.

\section{REFERENCES}

[1] C. Belmonte, D. Bocalleti and G. Pucacco, On the structure of the logarithmic potential, Astrophys. J. 669 (2007), 202-217.

[2] A. Buică, J. P. Françoise and J. Llibre, Periodic solutions of nonlinear periodic differential systems with a small parameter, Comm. on Pure and Appl. Anal. 6 (2007), 103-111.

[3] L. Jimenénez-LARA And J. Llibre, The cored and logarithm galactic potentials: Periodic orbits and integrability, J. Math. Phys. 53, 042901 (2012).

[4] E. A. Lacomba And J. Llibre, Dynamics of a galactic Hamiltonian system, J. Math. Phys. 53, 072901 (2012).

[5] I.G. MaLKIN, Some problems of the theory of nonlinear oscillations, (Russian) Gosudarstv. Izdat. Tehn.-Teor. Lit., Moscow, 1956.

[6] G. PuCACCO, Hamiltonian normal forms and galactic potentials, Chaos in Astronomy, Astrophys. Space Sci. Proc., Springer-Verlag, Berlin, 2009, pp. 137-149.

[7] G. Pucacco, D. Bocalleti and C. Belmonte, Periodic orbits in the logarithmic potential, Astron. Astrophys. 489 (2008), 1055-1063.

[8] G. Pucacco, D. Bocalleti and C. Belmonte, Quantitative predictions with detuned normal forms, Celes. Mech. Dyn. Astron. 102 (2008), 163-176. 
[9] M. Roseau, Vibrations non linéaires et théorie de la stabilité, (French) Springer Tracts in Natural Philosophy, Vol.8 Springer-Verlag, Berlin-New York, 1966.

${ }^{1}$ Departamento de Matemática, Universidade Federal Rural de Pernambuco, 52171 900, Recife, Pernambuco, Brasil

E-mail address: maite@dm.ufrpe.br

2 Departament de Matemàtiques, Universitat Autònoma de Barcelona, 08193 Bellaterra, Barcelona, Catalonia, Spain

E-mail address: jllibre@mat.uab.cat 